\title{
RHIC proton luminosity and polarization improvement
}

\author{
S.Y. Zhang
}

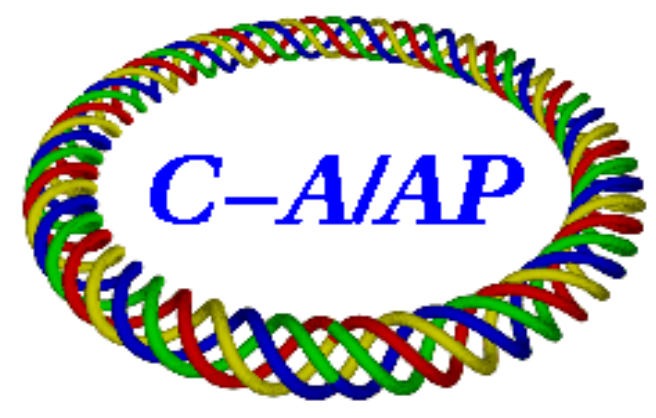

Collider-Accelerator Department Brookhaven National Laboratory Upton, NY 11973

Notice: This document has been authorized by employees of Brookhaven Science Associates, LLC under Contract No. DE-AC02-98CH10886 with the U.S. Department of Energy. The United States Government retains a nonexclusive, paid-up, irrevocable, world-wide license to publish or reproduce the published form of this document, or allow others to do so, for United States Government purposes. 


\title{
RHIC Proton Luminosity and Polarization Improvement
}

\author{
S.Y. Zhang * \\ January 17, 2014
}

\begin{abstract}
The RHIC proton beam polarization can be improved by raising the Booster scraping, which also helps to reduce the RHIC transverse emittance, and therefore to improve the luminosity. By doing this, the beam-beam effect would be enhanced. Currently, the RHIC working point is constrained between $2 / 3$ and $7 / 10$, the $2 / 3$ resonance would affect intensity and luminosity lifetime, and the working point close to $7 / 10$ would enhance polarization decay in store. Run 2013 shows that average polarization decay is merely $1.8 \%$ in 8 hours, and most fills have the luminosity lifetime better than 14 hours, which is not a problem. Therefore, even without beambeam correction, there is room to improve for RHIC polarization and luminosity. The key to push the Booster scraping is to raise the Booster input intensity, for that two approaches can be used. The first is to extend the LINAC tank 9 pulse width, which has been successfully applied in run 2006. The second is to raise the source temperature, which has been successfully applied in run 2006 and run 2012.
\end{abstract}

\section{Introduction}

The most important limiting factor of RHIC polarization is at the Booster scraping, where the scraping ratio is defined as the Booster input intensity/late intensity. Close correlation of the Booster scraping ratio and the RHIC H-jet polarization exist for $250 / 255 \mathrm{GeV}$ proton runs of 2011, 2012, and 2013. For comparable RHIC intensity, with the Booster scraping ratio of 1.5, the $\mathrm{H}$-jet polarization is below $50 \%$, and for ratios of 2 and 2.2, typical polarization is $52 \%$ and $55 \%$, respectively.

The most important limiting factor of RHIC transverse emittance is also at the Booster scraping. With larger Booster scraping ratio, the RHIC emittance tends to be smaller. This has been observed in RHIC proton runs, say, 2006, 2009, 2011, and 2012. The

${ }^{*}$ Acknowledgement: I would like to thank the encouragements and many helpful discussions for this work 
"magic" event in run 2013, where the RHIC transverse emittance is reduced at the time of AGS RF fix, is also related to the Booster scraping. The Booster scraping ratio is increased from 1.83 to 2.27 then.

By raising Booster scraping, the RHIC polarization can be improved. Meanwhile, the RHIC initial intensity and/or transverse emittance can be improved, which means the improvement of the initial luminosity. This implies that the RHIC beam-beam effect at the store would be enhanced. Currently, the working point is constrained between $2 / 3$ and $7 / 10.2 / 3$ is a strong $3 \mathrm{rd}$ order betatron resonance, which affects intensity lifetime, and $7 / 10$ is a spin depolarization resonance, the working point close to $7 / 10$ would enhance polarization decay in store. In run 2013, the last 40 fills have an average luminosity lifetime of 14.5 hours, which is comparable to previous runs, and is not a limiting factor. Also, typical polarization decay in run 2013 is about $2 \%$ in 8 hours of store, which is benign. Therefore, there is room to explore in terms of RHIC polarization and luminosity improvement.

The key factor for more Booster scraping is to raise the Booster input intensity. In addition to source and LINAC tuning, two approaches can be used. The first is to extend the LINAC tank 9 pulse width, which is currently limited at $300 \mu s$. In run 2006, it was extended to $400 \mu s$, with the improvement of RHIC polarization. The second approach is to raise the source $\mathrm{Rb}$ temperature, which has been successfully used in run 2006 and run 2012 for RHIC polarization improvement. The commissioning of the new polarized proton source in run 2013 has demonstrated improvement over the old source, more polarization improvement should be possible.

\section{RHIC polarization improvement}

The most important limiting factor of RHIC polarization is at the Booster scraping, where the Booster scraping ratio is defined as the Booster input intensity/late intensity. Despite many factors between the Booster to RHIC store, for example, the Booster to AGS transfer (BtA), the AGS ramping, the AGS to RHIC transfer (AtR), and the RHIC ramping and $\beta$ squeezing, striking correlations of the Booster scraping ratio and RHIC polarization has been presented in $100 \mathrm{GeV}$ polarized proton runs 2006, 2008, 2009 [1-4]. This correlation is similarly presented in $250 / 255 \mathrm{GeV}$ runs.

In Figure 1, the correlation between the Booster scraping ratio and the RHIC Yellow $\mathrm{H}$-jet polarization is shown for 250/255 GeV polarized proton runs 2011, 2012b, 2013. The Booster input intensity, Booster late intensity, and the RHIC bunch intensity are also presented. Some RHIC fills having problematic polarizations are marked by green dots: in run 2012b, it is due to RHIC snake current problem, and in run 2013 it is with the polarized proton ion source (OPPIS) vacuum problem. The Blue H-jet polarization is similar to the Yellow.

The bad polarization state is marked by red bars in both run 2011 and run 2013. With the Booster scraping ratio of 1.5 , the H-jet polarization is below $50 \%$. Good state is 

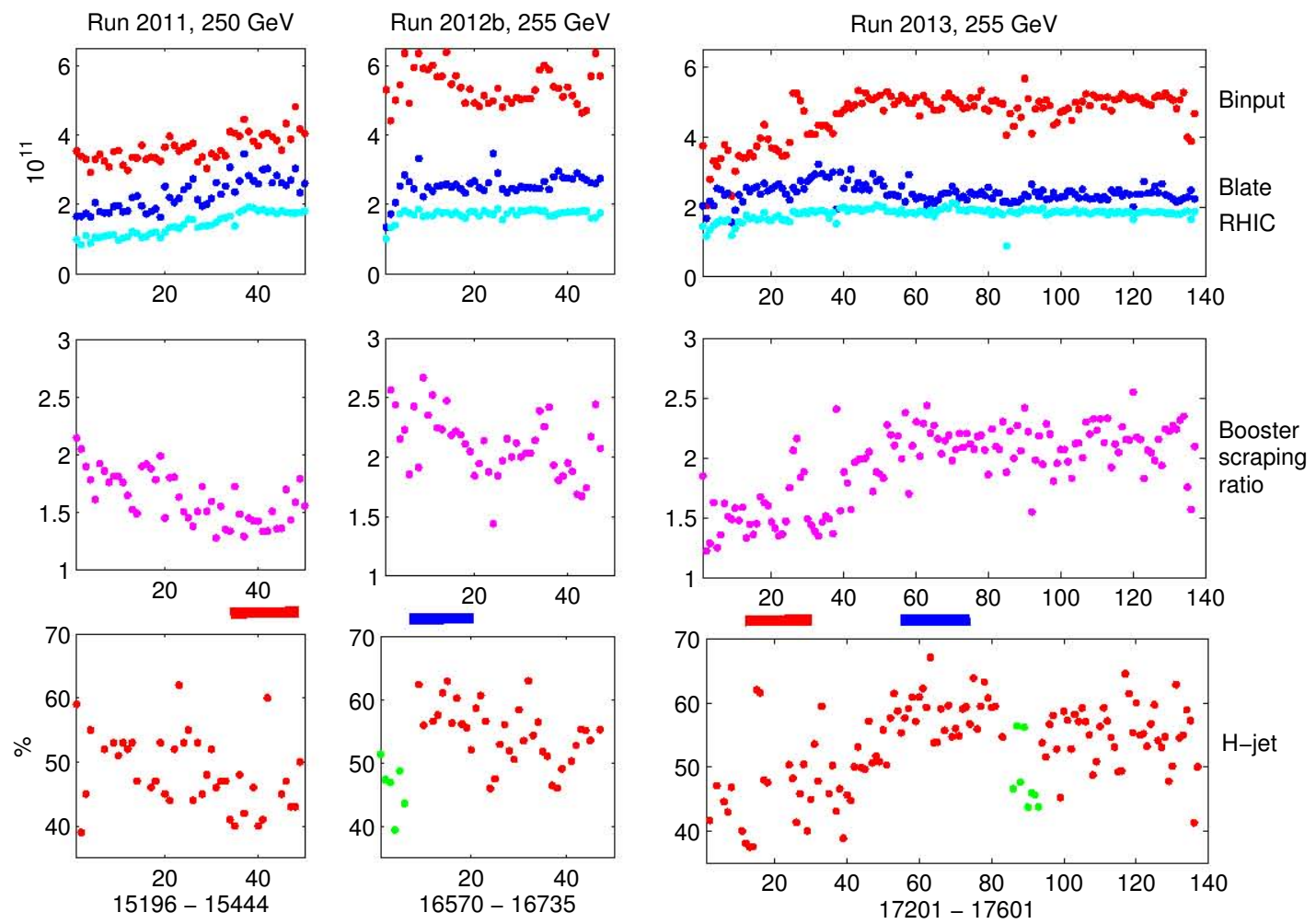

Figure 1: Striking correlation between the Booster scraping ratio and the RHIC Yellow H-jet polarization for 250/255 GeV polarized proton runs 2011, 2012b, 2013. Green dots in run 2012b are due to the RHIC snake current problem, and in run 2013 are with the OPPIS vacuum problem. Red bars are for bad state in both run 2011 and run 2013, where with the Booster scraping ratio 1.5 , the RHIC polarization is $<50 \%$. Blue bars are for good state in both run 2012b and 2013, where for Booster scraping ratio 2.3, the RHIC polarization is $>55 \%$. Note the similarities of the Booster scraping ratio and the polarization for bad state and good state, despite in different runs. 

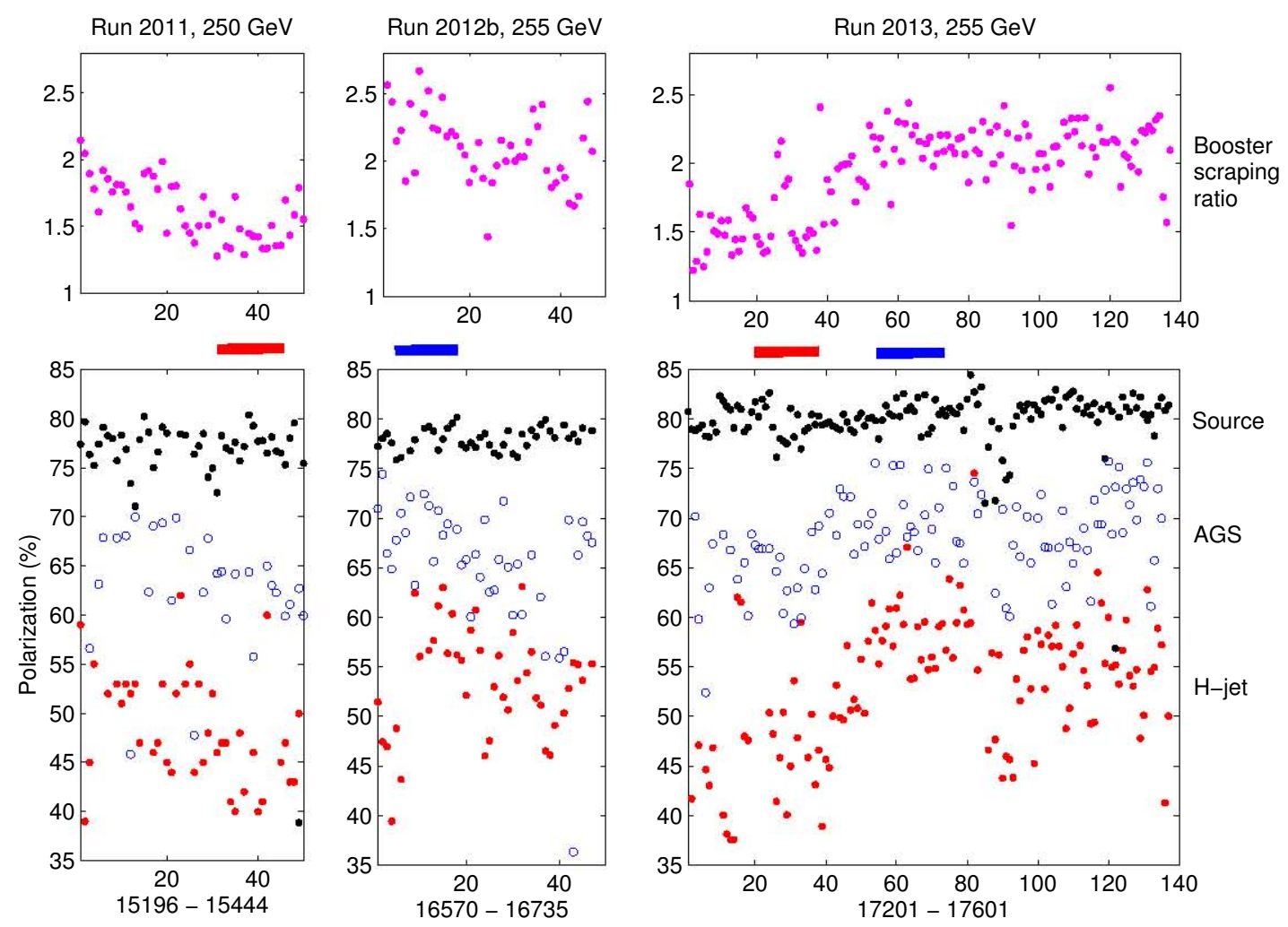

Figure 2: The OPPIS and AGS polarization measurement is added for $250 / 255 \mathrm{GeV}$ polarized proton runs 2011, 2012b, 2013. Striking correlations of Booster scraping ratio and the AGS polarization is shown. For bad state (red bars) of Booster scraping ratio of 1.5, the AGS polarization is between $60 \%$ to $65 \%$. For good state (blue bars) of Booster scraping ratio of 2.3 , the AGS polarization is around $70 \%$. OPPIS polarization in run 2013 is about $3 \%$ higher than run 2012b, but did not materialize in RHIC polarization. It is overwhelmed by the Booster scraping effect. 
marked by blue bars in both run $2012 \mathrm{~b}$ and run 2013. With the Booster scraping ratio of 2.2 to 2.3 , the $\mathrm{H}$-jet polarization is above $55 \%$.

In Figure 2, the OPPIS and the AGS polarization measurement is added for 250/255 GeV polarized proton runs 2011, 2012b, 2013. The striking correlations of Booster scraping ratio and the AGS polarization demonstrate that the effect of the Booster scraping is already shown in the AGS, not only in RHIC. For bad state (red bars) of Booster scraping ratio of 1.5, the AGS polarization is between $60 \%$ to $65 \%$. For good state (blue bars) of Booster scraping ratio of 2.3 , the AGS polarization is around $70 \%$.

The OPPIS polarization in run 2013 (new source) is about 3\% better than run 2011 and run 2012b, however, its effect is overwhelmed by the Booster scraping ratio, and did not present in RHIC polarization improvement.

In Figure 3, overall correlation between the Booster scraping ratio and the RHIC Yellow H-jet measurement in 250/255 GeV polarized proton runs 2011, 2012b, 2013 is shown. The best performance of run $2012 \mathrm{~b}$ and run 2013 are with the Booster scraping ration around 2.2, and the RHIC polarization is between $55 \%$ to $60 \%$. The general trend of RHIC polarization and the Booster scraping ratio indicates that with more Booster scraping, the RHIC polarization can be further improved.

In [1-4], detailed study of the Booster scraping effect on RHIC polarization is presented for $100 \mathrm{GeV}$ polarized proton run 2006, 2008, and 2009. In the successful run 2006, the Booster input intensity is raised to $>5 \times 10^{11}$, allowing high Booster scraping ratio, up to 2.8. With the RHIC bunch intensity of $>1.5 \times 10^{11}$ protons, the RHIC polarization at the injection is improved up to $65 \%$.

In $100 \mathrm{GeV}$ run 2009, the Booster input intensity is limited to $4 \times 10^{11}$, then as the RHIC bunch intensity is increased to $1.5 \times 10^{11}$, the Booster scraping ratio is declined from $>2$ to $<1.5$, resulted in the reduced RHIC polarization, below $55 \%$.

In Figure 4, the RHIC polarization vs. RHIC bunch intensity is shown for $100 \mathrm{GeV}$ run 2006 and run 2009. Along with the RHIC intensity improvement, the RHIC polarization in run 2006 and run 2009 is heading to different directions.

In run 2006, with the RHIC intensity improvement, the Booster scraping ratio is also increased. In run 2009, with the RHIC intensity increase, the Booster scraping ratio has to be reduced, resulted in the declining of RHIC polarization, all the way.

\section{RHIC transverse emittance improvement}

The most important limiting factor of RHIC transverse emittance is also at the Booster scraping. With more Booster scraping, the RHIC emittance tends to be smaller. This has been observed in RHIC proton runs, say, 2006, 2009, 2011, and 2012 [1-4].

The "magic" event in run 2013 [5], where with the AGS RF fix at the fill 17396 the $\mathrm{RHIC}$ transverse emittance is reduced, is also related to the Booster scraping ratio, which is increased at the time from 1.83 to 2.27 .

In Figure 5, the Booster scraping ratio is compared with the AGS vertical emittance 


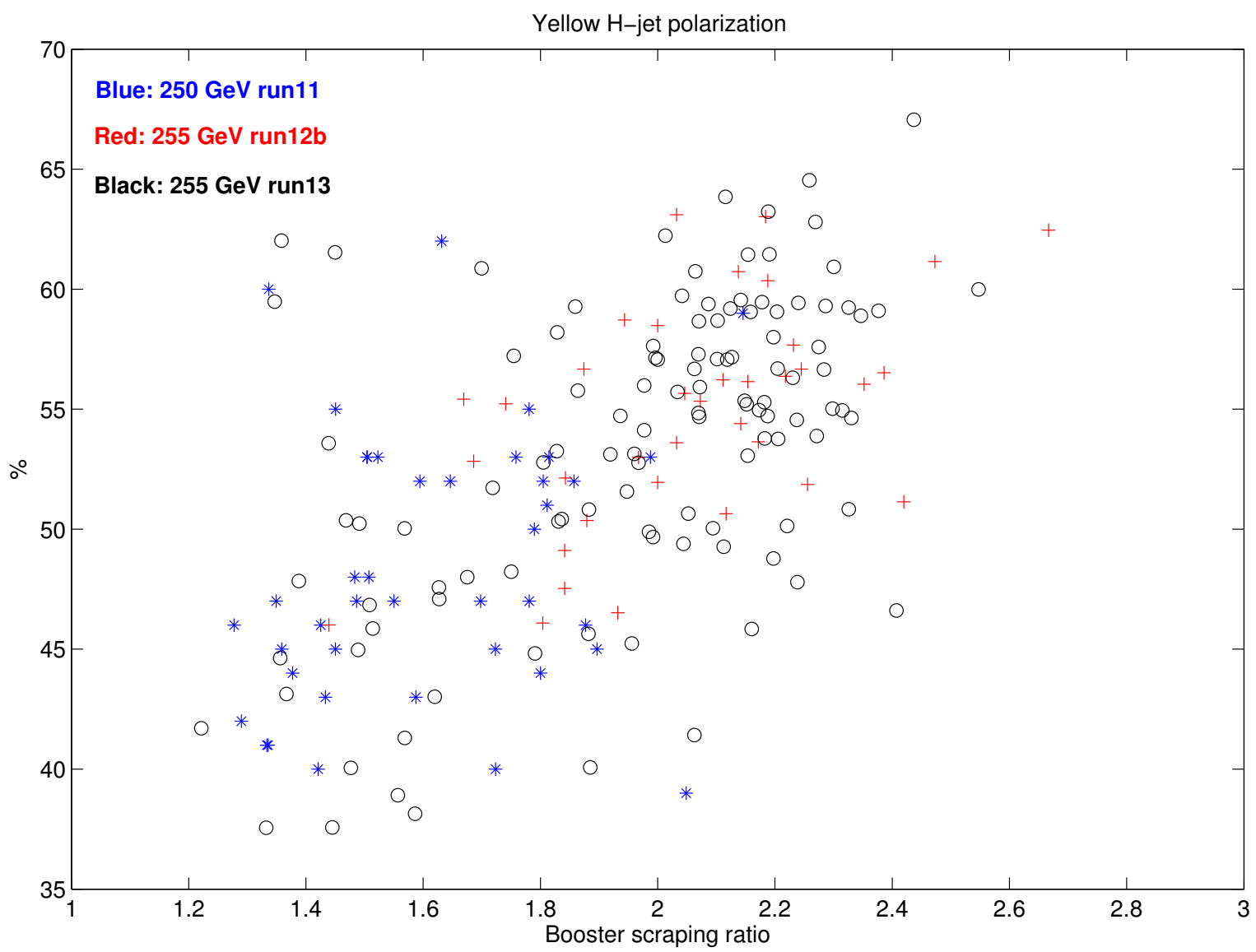

Figure 3: Overall correlation between the Booster scraping ratio and the RHIC Yellow Hjet measurement in 250/255 GeV polarized proton runs 2011, 2012b, 2013 demonstrates that the RHIC polarization can be further improved by pushing the Booster scraping ratio. Here the best performance of run $2012 \mathrm{~b}$ and run 2013 are with the Booster scraping ration around 2.2, and the RHIC polarization is between $55 \%$ to $60 \%$. Note that good RHIC polarization at low Booster scraping ratio is with low intensity, which is not shown. 

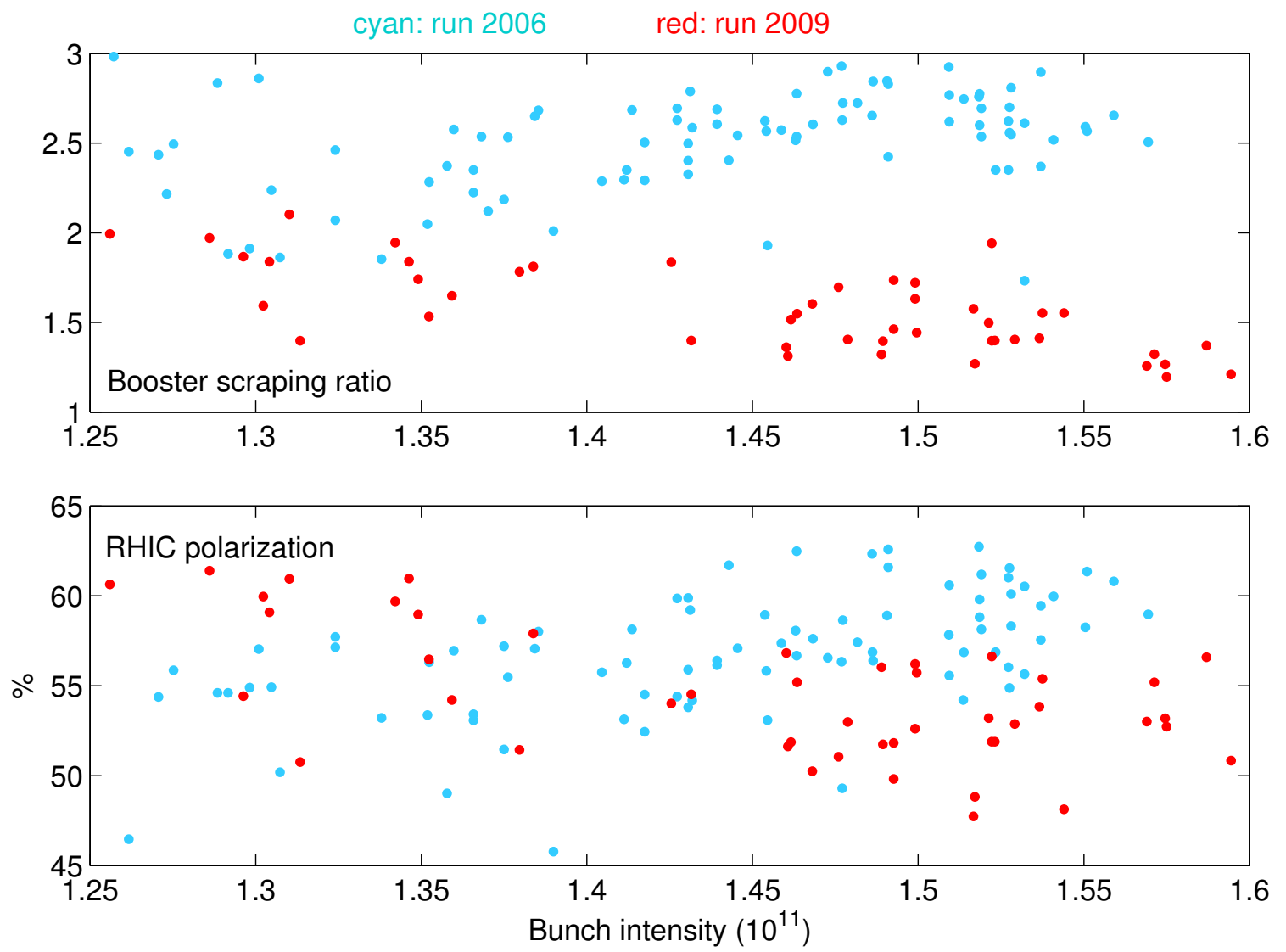

Figure 4: Booster scraping ratio and RHIC polarization vs. RHIC bunch intensity are shown for $100 \mathrm{GeV}$ run 2006 and run 2009. RHIC polarization in run 2006 is from Pol2006 yellow star.dat of Run06Offline, and in run 2009 it is from Pol2009 200gev yell.dat of Run09Offline. In run 2006, the Booster scraping is increased at high RHIC intensity, whereas in run 2009, since the Booster input intensity is limited, therefore, at high RHIC intensity, the Booster scraping has to be reduced, which caused RHIC polarization decline all the way. 
Run 2013. Booster scraping effect on AGS transverse emittance
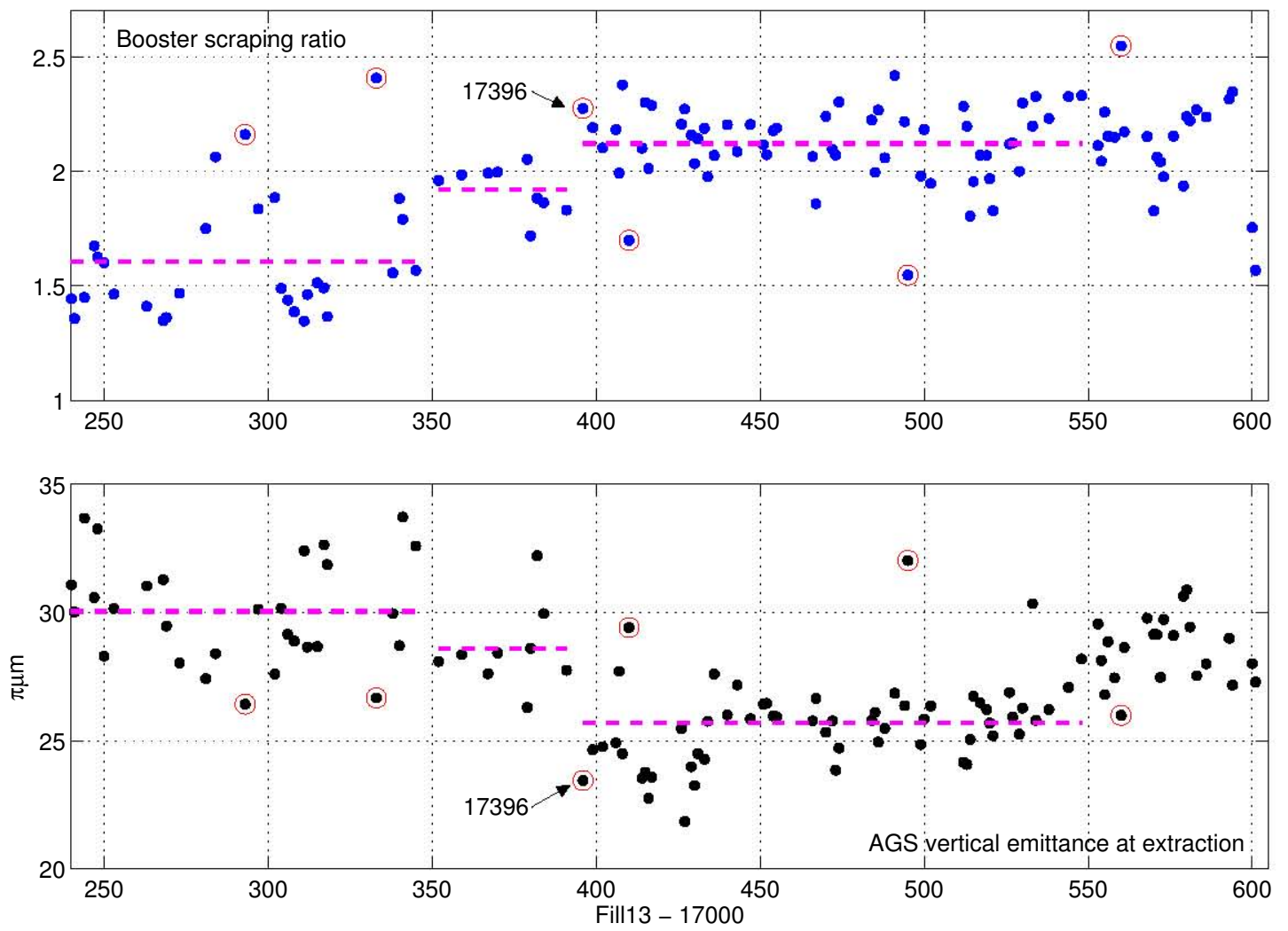

Figure 5: The Booster scraping ratio is compared with the AGS vertical emittance at the extraction for run 2013. There are 3 plateaus in the Booster scraping ratio, and also in the AGS vertical emittance, the averages are shown by the dashed lines. Fills of 17396, 17293, 17333, 17410, 17495, 17560, are marked by cycles in both the booster scraping ratio and the AGS emittance. The correlation between the Booster scraping ratio and the AGS transverse emittance is clearly demonstrated, not only by the 3 plateaus, but also by the 6 fills deviated from the plateaus 
Run 2013. Booster scraping effect on RHIC transverse emittance
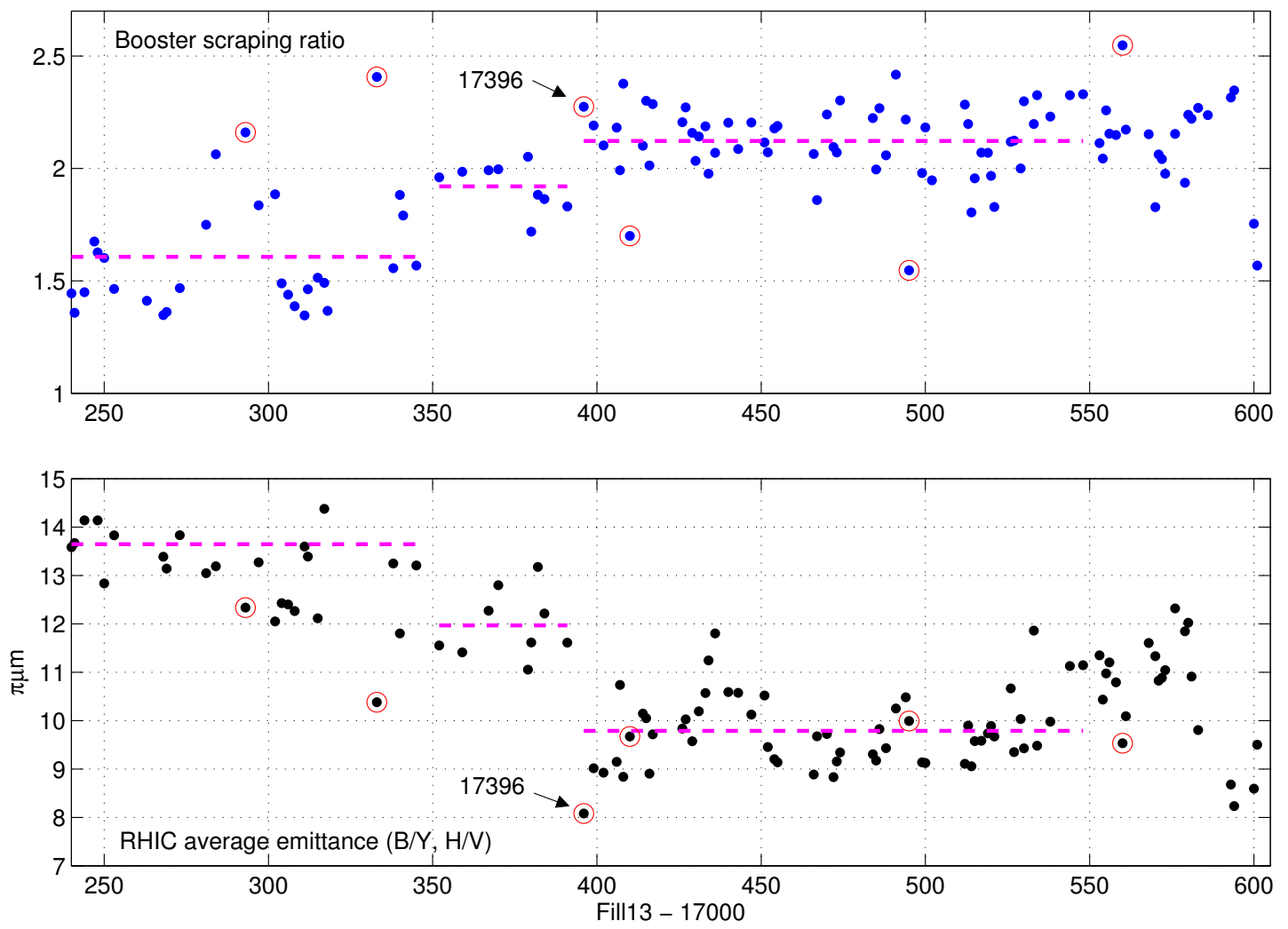

Figure 6: The Booster scraping ratio is compared with the IPM emittance measurement at the RHIC injection for run 2013, which is the average of Blue and Yellow, and horizontal and vertical. The 3 plateaus show the correlation between the Booster scraping ratio and the RHIC average transverse emittance. The correlation between the Booster scraping ratio and the RHIC transverse emittance is clearly demonstrated by the 3 plateaus. Nevertheless, for the 6 marked fills, the correlation is not as consistent as the AGS emittance, shown in Figure 5. This demonstrates why an individual study might be misleading. 
at the extraction for run 2013 [6]. There are 3 plateaus in the Booster scraping ratio, i.e., fills 17240-17345, fills 17352-17391, fills 17396-17548, and the average of each plateau is shown by the dashed lines. The average AGS vertical emittance, shown by dashed lines in these 3 plateaus, demonstrate the effect of the Booster scraping on the AGS emittance. Moreover, the "magic" fill of 17396 and other 5 arbitrarily chosen fills that deviate from the plateaus, 17293, 17333, 17410, 17495, 17560, are marked by red cycles in both the booster scraping ratio and the AGS emittance. The correlation is very clearly demonstrated.

In Figure 6, the Booster scraping ratio is compared with the IPM emittance measurement at the RHIC injection for run 2013 [6]. The IPM emittance is the average of Blue and Yellow, both horizontal and vertical. The 3 plateaus show the correlation between the Booster scraping ratio and the RHIC average transverse emittance. Nevertheless, from the 6 marked fills, the RHIC emittance is not as consistently correlated with the Booster scraping ratio, see Figure 5 for the AGS vertical emittance. This demonstrates the complication in an individual study to identify the Booster scraping effect. As usual, the general trend of the emittance with respect to the Booster scraping is more dependable. An individual study of the Booster scraping, even with both AGS and RHIC, could be misleading.

In Figure 7, overall relations between the Booster scraping ratio, the AGS emittance, the RHIC emittance and the H-jet polarization are shown. In general, the lower Booster scraping ratio implies larger emittance, and lower polarization. With larger Booster scraping ratio, the machine performance is better.

Fills after 17550 are less consistent in the correlations, compared with the 3 plateaus. One possible reason is that during that period, a part of the Booster scraping is used for horizontal, and it is known that the vertical scraping is more effective in polarization improvement [1-3].

At 17333, the RHIC is switched from the e-lens lattice back to run $2012 \mathrm{~b}$ lattice. The difference between the two lattices cannot be clearly identified in this plot.

The dependence of the RHIC transverse emittance on the Booster scraping has been presented in [1-3] for $100 \mathrm{GeV}$ run 2006 and run 2009. In run 2009, since the Booster input intensity is limited at $4 \times 10^{11}$, with the higher RHIC intensity, the Booster scraping has to be reduced, which resulted in larger transverse emittance.

In specific, when the RHIC bunch intensity is increased from $1.3 \times 10^{11}$ to $1.6 \times 10^{11}$ in run 2009, the Booster scraping ratio is reduced from $>2$ to $<1.5$, and the transverse emittance increased from $13 \pi \mu m$ to $18 \pi \mu m$. The consequence is as reported by the run coordinator in [7]: "Integrated luminosity increased almost linearly during the entire run, the first week was already as good as any other week".

In Figure 8, the Booster scraping ratio in $100 \mathrm{GeV}$ run 2006 and run 2009 are compared with the RHIC transverse emittance. During the RHIC intensity push, the Booster scraping is reduced in run 2009, together with the transverse emittance rising all the way. The run 2006, however, shows that with the increased Booster scraping, the transverse emittance is almost flat with the intensity increase. 


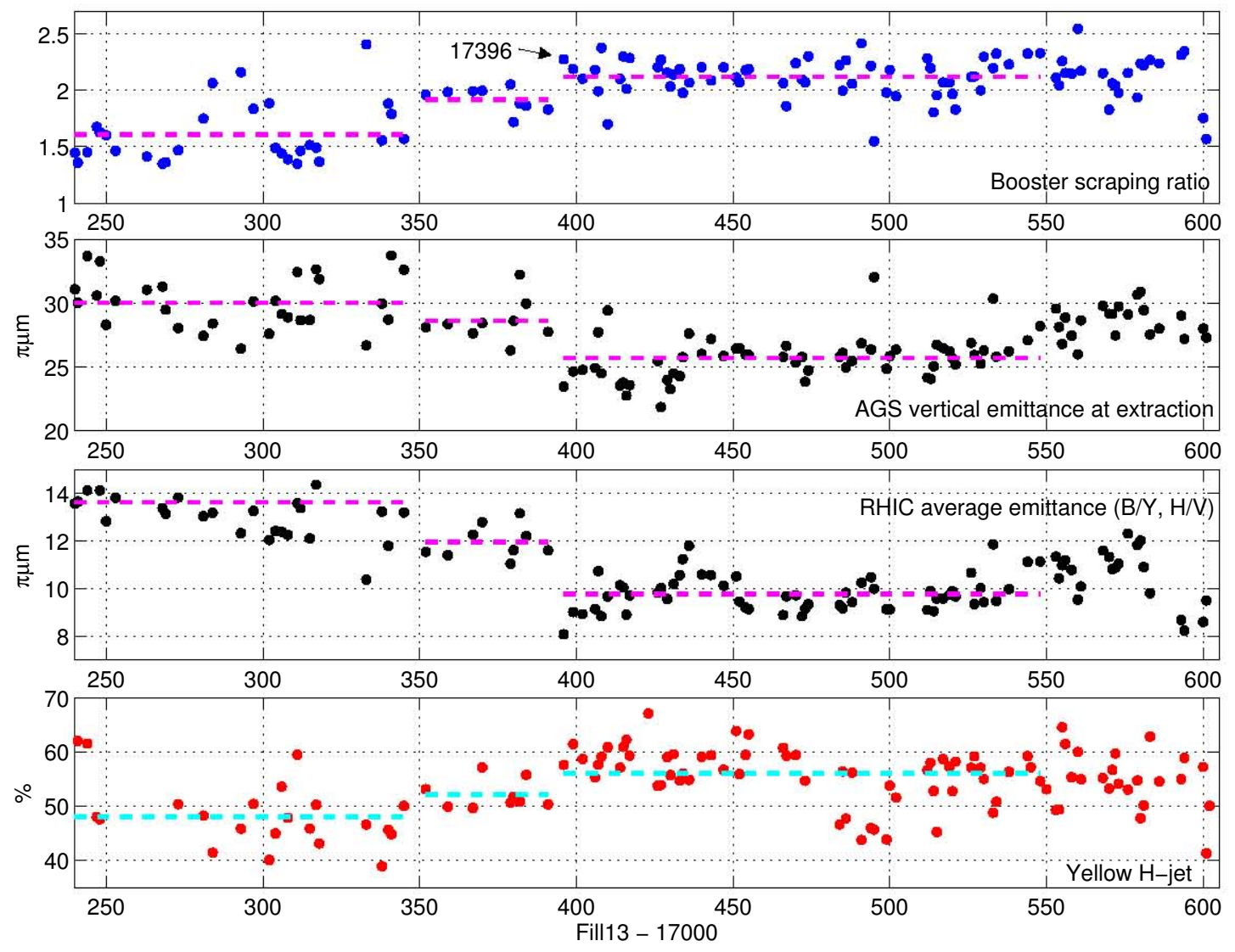

Figure 7: Overall relations between the Booster scraping ratio, the AGS transverse emittance, the RHIC transverse emittance, and the H-jet polarization are shown. The dashed lines are the average values. The Booster scraping after 17550 is partially used for horizontal, which might be less effective for emittance and polarization improvement. In general, the lower Booster scraping ratio implies larger emittance at both AGS and RHIC, and also lower RHIC polarization. With larger Booster scraping ratio, the machine performance is improved. 
cyan: run $2006 \quad$ red: run 2009
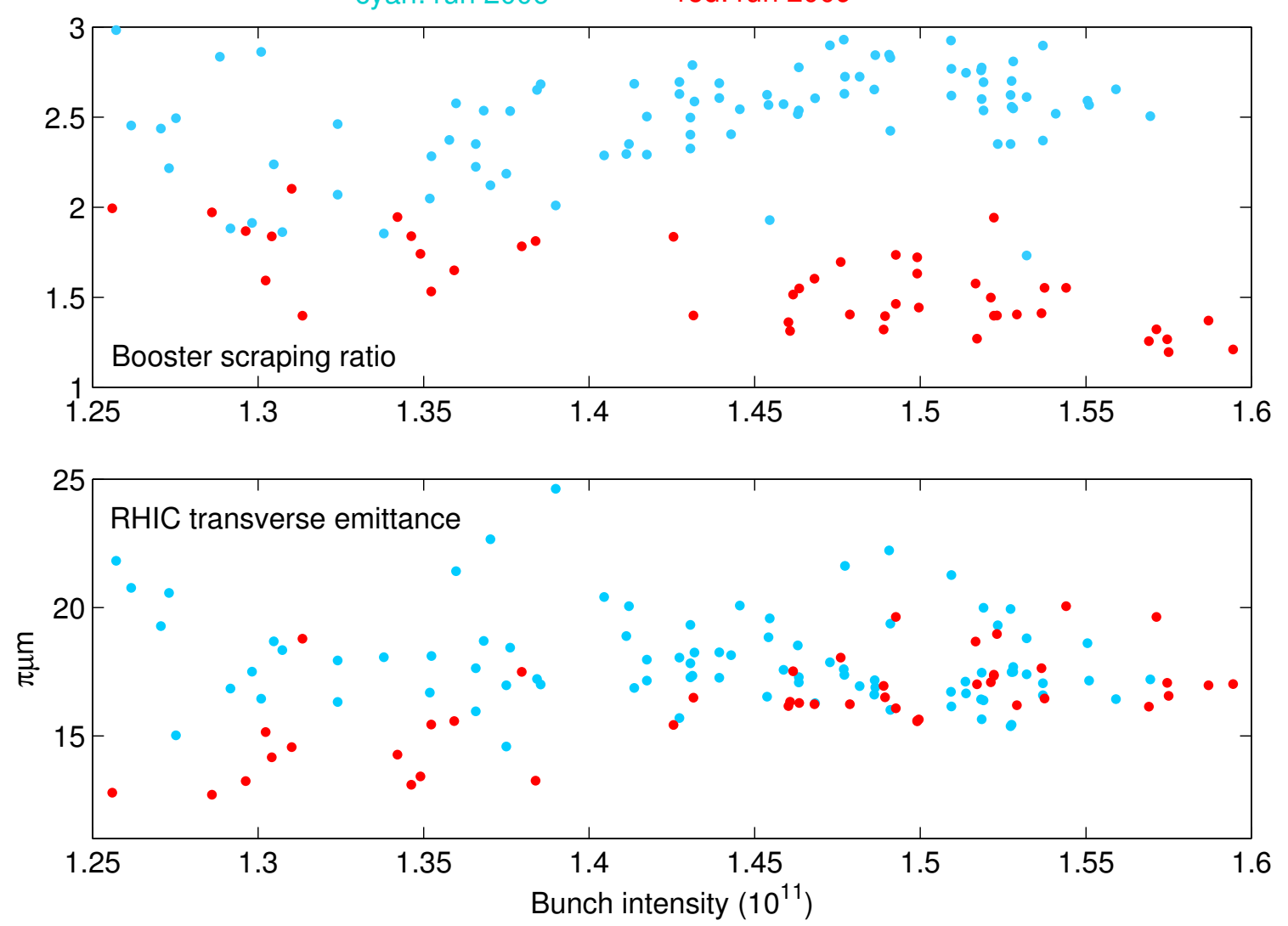

Figure 8: Booster scraping ratio and RHIC transverse emittance vs. RHIC bunch intensity are shown for $100 \mathrm{GeV}$ run 2006 and run 2009. The RHIC emittance is calculated from PHENIX ZDC at early collision, without corrections. In run 2006, the Booster scraping is increased at high RHIC intensity, whereas in run 2009, since the Booster input intensity is limited, therefore, at high RHIC intensity, the Booster scraping has to be reduced, which caused RHIC transverse emittance rising all the way. The larger emittance at higher RHIC intensity in run 2009 is the reason why "integrated luminosity increased almost linearly during the entire run". 


\section{Working point limitations}

By raising the Booster scraping ratio, both RHIC polarization and RHIC transverse emittance can be improved. On the other hand, the beam-beam effect at the collision would be enhanced.

Currently, the RHIC working point is constrained between $2 / 3$ to $7 / 10$, the $2 / 3$ resonance would affect intensity lifetime, and the working point close to $7 / 10$ would enhance polarization decay. The observation in run 2013 shows that, with the current beam-beam parameter, there is a room of the proton luminosity and/or polarization improvements.

In Figure 9, the Yellow polarization decay of 17328 to 17602 in run 2013 [8] and the luminosity lifetime of the corresponding fills are shown.

The Yellow polarization decay shown is from Y1D, which has the average polarization decay of $0.083 \%$ /hour, or an absolute loss of polarization of $0.66 \%$ in 8 hours of store, which is benign. Similarly, the polarization decay rate is $0.189 \% /$ hour for Y2U, also it is $0.355 \%$ /hour for B1U and 0.276\%/hour for B2D. In overall, the average polarization loss for Yellow and Blue is $1.8 \%$ in 8 hours of store.

In fact, the overall average polarization loss for Yellow and Blue has been $6.3 \%$ in 8 hours in $255 \mathrm{GeV}$ run 2012b [8]. Nevertheless, in overall, the reported average polarizations in run $2012 \mathrm{~b}$ and run 2013 are the same, at 52\%. This shows that the polarization decay is not a limiting factor in the current runs, and there is some room of the working point toward the direction of $7 / 10$.

Regarding to other direction, toward $2 / 3$, it has been observed that if the working point is too low, then for larger beam-beam parameter, the $2 / 3$ resonance can cause significant beam loss. The result is the reduced intensity lifetime, and the lower luminosity lifetime.

For example, in run 2011 at fill 15386, the Blue/Yellow vertical tunes are raised by $0.002 / 0.003$, respectively, and the luminosity lifetime is improved from about 9 hours to 16 hours. The integrated luminosity of 15386 is improved to $2.38 p b^{-1}$, from the previous best fill of 15337 at $1.56 p b^{-1}$. The 10 long fills just before 15386 is averaged at $1.28 p b^{-1}$, with that, the improvement of the integrated luminosity of 15386 is $86 \%$.

As shown in Figure 9, the luminosity lifetime is not a limiting factor in entire run 2013. In fact, the average luminosity lifetime of the last 40 fills in run 2013 is 14.5 hours.

In [9], the RHIC beam-beam effect on the transverse emittance growth and the luminosity lifetime is reported. In [3], it is shown that at the beam-beam parameter of 0.01 , the typical luminosity lifetime is 15 hours. It can be projected that at the beam-beam parameter of 0.02 , the luminosity lifetime would be as low as 7 hours. Then, the correction of beam-beam effect, e.g., by e-lens, is needed for the enhanced beam-beam effect.

The luminosity lifetime of more than 14 hours in run 2013 has shown that to raise the Booster scraping, and hence to reduce the RHIC transverse emittance, even with the enhanced beam-beam effect, is a right choice in the luminosity improvement. Note that the benefit of the more Booster scraping can be applied to reduce the transverse emittance with same intensity, and/or to raise intensity with less emittance increase.

In Figure 10, the run 2013 "magic" event fill 17396 is compared with 17391, which is 

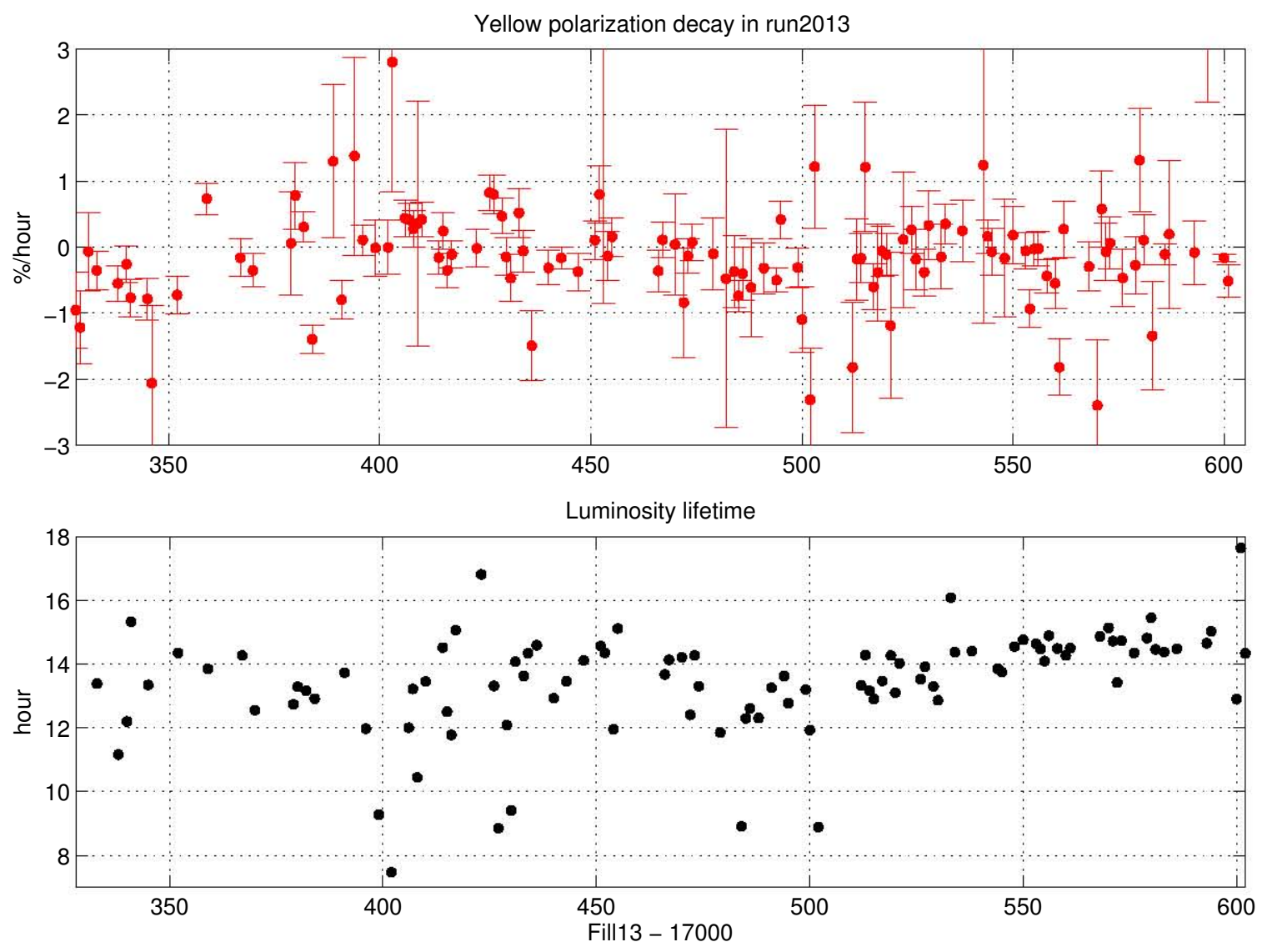

Figure 9: The Yellow polarization decay of 17328 to 17602 in run 2013 is on the top, which is from Y1D. The decay shown in Y2U, B1D, B2U are all the similar. In overall, the averaged polarization decay in run 2013 is $1.8 \%$ in 8 hours of store, which is benign. On the bottom is the average luminosity lifetime from 1.5 to 5.5 hours after accramp. In the later run 2013, it is better than 14 hours, and therefore it is not a problem. This plot shows that, in run 2013, there is a room of the enhanced beam-beam parameter in terms of the working point between 2/3 and 7/10. With this room, the luminosity and/or polarization can be improved from the current state. 
Fill 17391
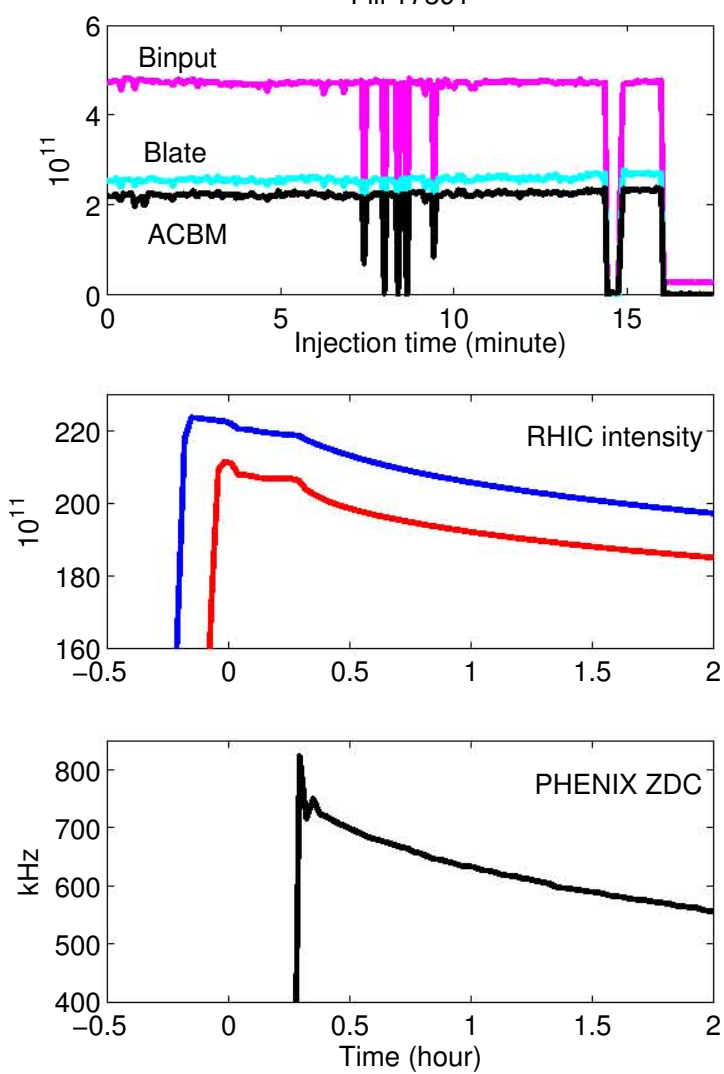

Fill 17396
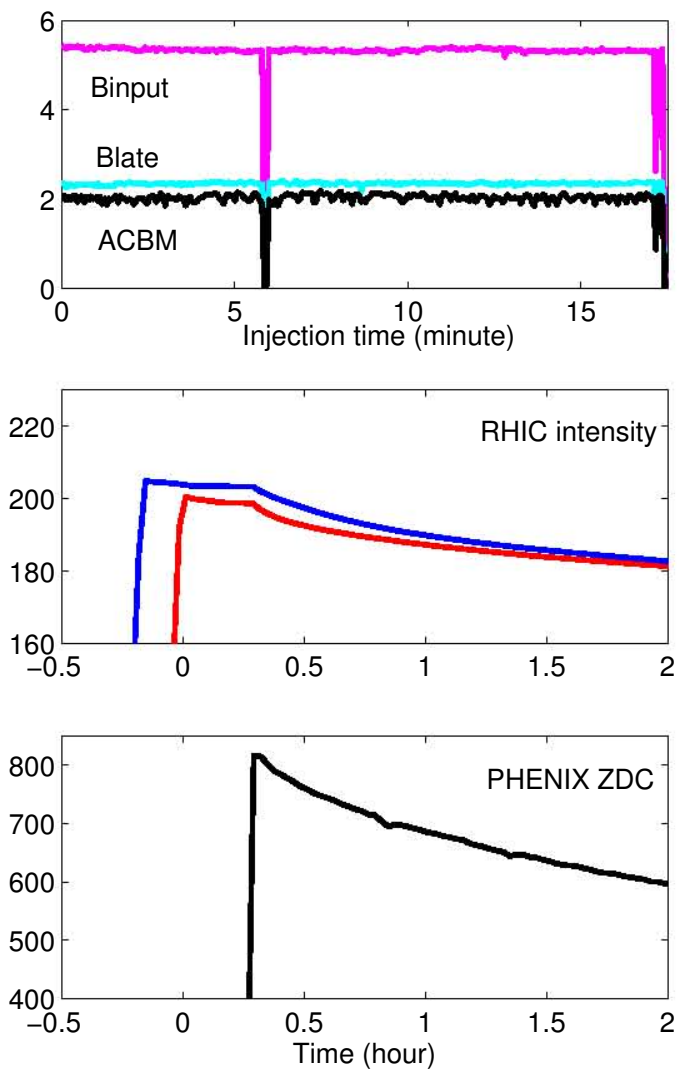

Figure 10: The "magic" event fill 17396 in run 2013 is compared with 17391, which is the long fill just before 17396. With the higher Booster input intensity, and therefore more Booster scraping, the fill 17396 had significant luminosity improvement from 17391. Not shown is that 17396 also had $14.5 \%$ improvement in Yellow H-jet polarization over $17391,57.6 \%$ vs. $50.3 \%$, which is also due to the increased Booster scraping. At the same time, the beam-beam parameter of 17391 and 17396 are almost the same. This plot shows that the big improvement of both luminosity and polarization of the "magic event" 17396 comes from the increased Booster scraping. 
the long fill just before 17396, to illustrate the RHIC proton luminosity and polarization improvement.

The Booster input and late intensities, the AGS and RHIC intensities, and the PHENIX ZDC are shown. The Booster input intensity is increased from $4.76 \times 10^{11}$ of 17391 to $5.30 \times 10^{11}$ of 17396 , and the Booster late is decreased from $2.60 \times 10^{11}$ to $2.33 \times 10^{11}$, which implies that the Booster scraping ratio is increased from 1.83 in 17391 to 2.27 in 17396.

The average bunch intensity at the RHIC collision is in fact reduced from $1.91 \times 10^{11}$ of 17391 to $1.80 \times 10^{11}$ of 17396 , with that, the PHENIX ZDC is increased from $745 \mathrm{kHz}$ of 17391 to $820 \mathrm{kHz}$ of 17396 . This is due to the reduced transverse emittance of 17396 , which is, in turns, due to the increased Booster scraping ratio.

Not shown in Figure 10 is that Yellow H-jet polarization of 17391 is $50.3 \%$, whereas it is $57.6 \%$ in 17396 , a $14.5 \%$ improvement. This is again due to the increased Booster scraping ratio.

From the simple relation of the intensity and the ZDC, which is inversely proportional to the transverse emittance, the beam-beam parameters of 17391 and 17396 are almost the same, indicating that the key factor in the "magic" event is the Booster scraping, rather than the beam-beam.

A brief summary of the comparison of 17391 and 17396 is presented in Table 1.

\begin{tabular}{ccc||c} 
& 17391 & 17396 & $17396 / 17391, \%$ \\
\hline \hline Booster input intensity, $10^{11}$ & 4.76 & 5.30 & 111.3 \\
\hline Booster late intensity, 10 & 2.60 & 2.33 & 89.6 \\
\hline AGS CBM intensity, 10 ${ }^{11}$ & 2.26 & 2.05 & 90.7 \\
\hline RHIC bunch intensity, 10 & 1.91 & 1.80 & 94.2 \\
\hline Booster scraping ratio & 1.83 & 2.27 & 124.0 \\
\hline PHENIX ZDC, $k H z$ & 747 & 820 & 109.8 \\
\hline Yellow H-jet, $\%$ & 50.3 & 57.6 & 114.5
\end{tabular}

Table 1. Compare 17391 with the "magic event" 17396. With the increase of the Booster scraping ratio by 24\%, 17396 has $9.8 \%$ and $14.5 \%$ improvement over 17391 for PHENIX ZDC and Yellow H-jet polarization, respectively. Note that the beam-beam parameter of 17396 and 17391 is about the same.

\section{Key factor for RHIC improvement}

The key factor in raising the Booster scraping ratio is to raise the Booster input intensity. In addition to source and LINAC tuning, two approaches can be used.

The first approach is to extend the LINAC tank 9 pulse width.

In run 2006, the tank 9 pulse width was extended to $400 \mu s$, which allowed the Booster input intensity to be as high as $>5 \times 10^{11}$, and the Booster scraping ratio better than 
2.5. As the result, even with the increase of RHIC bunch intensity, $>1.5 \times 10^{11}$, the polarization at RHIC injection is improved up to $65 \%$, as shown in [1-4].

Starting from early 2009, the tank 9 pulse width is limited at $300 \mu s$. Therefore, the Booster input intensity is limited at $4 \times 10^{11}$ in the entire run 2009. With the RHIC intensity push, the Booster scraping ratio was reduced from $>2$ to lower than 1.5. As the result, the RHIC polarization was reduced and the RHIC transverse emittance was increased at high intensity.

The comparison of run 2009 and run 2006 has clearly demonstrated the effect of the Booster scraping with the RHIC polarization and emittance, as shown in Figure 4 and Figure 8.

It is worth to mention that the tank 9 pulse shape is not perfect, and therefore using wider pulse implies both lower average polarization and lower average intensity at the tank 9. Nevertheless, in overall, with the wider tank 9 pulse, hence the higher Booster input intensity, more Booster scraping can be applied, the RHIC performance is improved.

The second approach is to raise the source $\mathrm{Rb}$ temperature.

In later run 2006, the source Rb temperature was raised to $>100$ degrees, which helped, together with the extended tank 9 pulse width, for Booster input intensity increase. The source temperature was not raised for run 2009 and the $250 \mathrm{GeV}$ run 2011. In run 2012, the source $\mathrm{Rb}$ temperature was again raised to $>100$ degrees, leading to higher Booster input intensity and better Booster scraping. The run 2012 was successful in terms of the improved polarization and luminosity.

It is also worth to mention that with the higher $\mathrm{Rb}$ temperature, the source polarization is reduced significantly. For example, in a study of early 2013 with the new OPPIS, when the $\mathrm{Rb}$ temperature is increased from 80 degrees to 100 degrees, the tank 9 current is increased from $180 \mu \mathrm{A}$ to $320 \mu \mathrm{A}$ (with the $85 \%$ transmission efficiency from tank 9 to the Booster, this implies that the Booster input intensity is increased from $2.9 \times 10^{11}$ to $\left.5.1 \times 10^{11}\right)$. At the same time, the source polarization is reduced from $83 \%$ to $76 \%$.

Similar performance is actually observed with the old source. For example, at the injection of the golden fill 16697 in run 2012b, the source temperature is raised from 90 degree to 100 degree, the Booster input intensity is increased from $4 \times 10^{11}$ to $5 \times 10^{11}$, but the source polarization is reduced from $>80 \%$ to about $77 \%$. In fact, $77 \%$ is the typical source polarization at the RHIC filling period of time in entire run $2012 \mathrm{~b}$, rather than $>80 \%$ polarization that the source was able to provide. Despite this, the run 2012b was a successful run in terms of RHIC polarization, with an improvement over run 2011.

In general, what observed in previous runs is that with the higher source Rb temperature, the source polarization is lower, but since better Booster scraping can be applied, in overall, the RHIC polarization is improved. 


\section{Conclusion}

The RHIC proton beam polarization can be improved by raising the Booster scraping ratio, which also helps to reduce the RHIC transverse emittance, and therefore to improve the RHIC luminosity.

By doing this, the beam-beam effect would be enhanced.

Currently, the RHIC working point is constrained between $2 / 3$ and $7 / 10$, touching $7 / 10$ may enhance polarization decay, and touching $2 / 3$ may reduce intensity lifetime, and hence the luminosity lifetime.

Run 2013 shows that the average polarization decay is merely $1.8 \%$ in 8 hours, which is benign, and the later fills has the luminosity lifetime better than 14 hours, which is not a problem. Therefore, it is clear that there is a room to increase the RHIC polarization and luminosity with the Booster scraping push, even with the enhanced beam-beam effect.

The key to push the Booster scraping is to raise the Booster input intensity. To extend the LINAC tank 9 pulse width and to raise the source Rb temperature has been successfully applied in run 2006 and run 2012.

With the better Booster scraping, the RHIC luminosity and polarization should be improved. The new OPPIS has demonstrated higher source polarization and intensity than the old one, therefore, one expects even more RHIC improvement.

\section{References}

[1] "Polarization Issues in Run 2008", S.Y. Zhang, L. Ahrens, H. Huang, K. Zeno, C$\mathrm{A} / \mathrm{AP} / 316,7 / 1 / 2008$

[2] "Effects of Booster Scraping in Polarized Proton Runs 2006 and 2008", S.Y. Zhang, L. Ahrens, H. Huang, K. Zeno, C-A/AP/338, 1/1/2009

[3] "Luminosity Issues in $2009100 \mathrm{GeV}$ Polarized Proton Run", S.Y. Zhang, C$\mathrm{A} / \mathrm{AP} / 361,8 / 1 / 2009$

[4] "Some Luminosity Issues in Polarized Proton Runs", S.Y. Zhang, H. Huang, V. Schoefer, unpublished notes, $11 / 30 / 2012$

[5] "2013 p-p Run: RHIC Summary", V. Ranjbar, RHIC Retreat 2013

[6] "Managing Emittances: Transverse and Longitudinal", V. Schoefer, RHIC Retreat 2013

[7] "Run 9: $100 \mathrm{GeV",} \mathrm{C.} \mathrm{Montag,} \mathrm{RHIC} \mathrm{Retreat} 2009$

[8] "RHIC Polarization Improvement: Status ", M.Bai, RHIC Retreat 2013

[9] "Proton beam emittance growth in the Relativistic Heavy Ion Collider", S.Y. Zhang and V. Ptitsyn, Phys. Rev. ST Accel. Beams 11, 051001, 2008 